\title{
Iterative Overlap QRM-ML Block Detection for Single-Carrier MIMO Transmission Without CP Insertion
}

\author{
Hideyuki Moroga • Tetsuya Yamamoto • \\ Fumiyuki Adachi
}

Published online: 7 January 2014

(C) The Author(s) 2014. This article is published with open access at Springerlink.com

\begin{abstract}
QR decomposition and M-algorithm based near maximum likelihood block detection (QRM-MLBD) significantly improves the single-carrier (SC) multiple-input multipleoutput (SC-MIMO) transmission performance in a frequency-selective fading channel. In the conventional QRM-MLBD, the cyclic prefix $(\mathrm{CP})$ is inserted in order to avoid the inter-block interference (IBI). However, CP insertion reduces the transmission efficiency. In this paper, an iterative overlap QRM-MLBD is proposed for SC-MIMO transmission with no CP insertion. It is confirmed by computer simulation that the iterative overlap QRM-MLBD with no $\mathrm{CP}$ insertion provides improved throughput performance while reducing the computational complexity over the conventional QRM-MLBD with CP insertion.
\end{abstract}

Keywords Single-carrier $\cdot$ MIMO $\cdot$ No cyclic prefix $\cdot$ QR decomposition $\cdot$ M-algorithm

\section{Introduction}

In the next generation mobile communication systems, broadband data services are demanded. Multiple-input multiple-output (MIMO) spatial multiplexing [1] provides broadband data transmissions without increasing the signal bandwidth. For uplink (mobile terminal to base-station) application, single-carrier (SC) transmission is suitable because of its lower peak-to-average power ratio (PAPR) property $[2,3]$ compared with multi-carrier transmission, e.g., orthogonal frequency division multiplexing (OFDM) [4]. Thus, SC-MIMO has

\footnotetext{
H. Moroga $\cdot$ T. Yamamoto $(\varangle) \cdot$ F. Adachi

Department of Communications Engineering, Graduate School of Engineering,

Tohoku University, 6-6-05 Aza-Aoba, Aramaki, Aoba-ku,

Sendai 980-8579, Japan

e-mail: yamamoto@mobile.ecei.tohoku.ac.jp

H. Moroga

e-mail: moroga@mobile.ecei.tohoku.ac.jp

F. Adachi

e-mail: adachi@ecei.tohoku.ac.jp
} 
been adopted for the uplink transmission in 3rd generation partnership project long term evolution-advanced (3GPP LTE-A) systems [5].

For broadband signal transmission, wireless channel is severely frequency-selective [6]. The broadband SC-MIMO spatial multiplexing suffers from inter-symbol interference (ISI) arising from the severe frequency-selectivity of the channel. The use of the cyclic prefix (CP) and frequency-domain block detection such as a computational efficient minimum mean square error (MMSE) based linear detection [7] can improve the transmission performance of SC-MIMO spatial multiplexing. However, a big performance gap from the maximum likelihood (ML) performance still exists due to the presence of residual ISI and inter-antenna interference (IAI). Recently, QR decomposition and M-algorithm based near-maximum likelihood block detection (QRM-MLBD) was proposed [8,9] for broadband SC-MIMO spatial multiplexing. QRM-MLBD significantly improves the transmission performance of SC-MIMO spatial multiplexing in a frequency-selective fading channel while significantly reducing the computational complexity compared with ML detection.

The conventional block detection schemes, such as MMSE based linear detection and QRM-MLBD, require the insertion of CP to avoid the inter-block interference (IBI). However, $\mathrm{CP}$ insertion reduces the transmission efficiency. As a linear detection, overlap frequencydomain linear detection was proposed [10-12] in which the received symbol stream is divided into a sequence of blocks of $X$ symbols each and then, frequency-domain block detection is applied to an extended block of $N_{c}$ symbols centering the $X$-symbol block of interest ( $X \leq N_{c}$ ). Note that $N_{c}$-symbol block is first transformed by the discrete Fourier transform (DFT) into the frequency-domain signal. Knowing that the residual IBI is significant near both ends of $N_{c}$-symbol block after MMSE based linear detection, only $X$ symbols are picked up in order to avoid the residual IBI. However, a big performance gap from the ML performance is observed due to insufficient suppression of interferences, i.e., IAI, ISI, and also IBI.

In $[13,14]$, we presented the SC transmission using time-domain iterative overlap QRMMLBD with no CP insertion for single-input single-output (SISO) systems. In overlap QRMMLBD, the concept of overlap processing is applied to QRM-MLBD, in which the received symbol stream is divided into a sequence of blocks of $X$ symbols each and then, QRMMLBD is applied to an extended block of $N_{c}+L-1$ symbols to detect an $N_{c}$-symbol block including the $X$-symbol block of interest at the beginning, where $L$ denotes the channel length in symbols (in this paper, the channel is assumed to be composed of symbol-spaced $L$ propagation paths). The residual IBI is significant near the end of $N_{c}$-symbol block after QRM-MLBD. Based on the above observation, only $X$ symbols are picked up in order to avoid the residual IBI. To improve the IBI suppression, iterative processing and IBI cancellation are also introduced. Note that the proposed iterative overlap QRM-MLBD is implemented in the time-domain (no DFT is used in block detection). This is because time-domain overlap QRMMLBD is equivalent to the frequency-domain overlap QRM-MLBD [13] and time-domain processing has an advantage in terms of the computational complexity.

In this paper, we extend the previously proposed iterative overlap QRM-MLBD to SC-MIMO spatial multiplexing with no CP insertion. To extend our previously proposed algorithm to the MIMO systems, we introduce an appropriate modification of the received signal vector for SC-MIMO spatial multiplexing. It is confirmed by computer simulation that the iterative overlap QRM-MLBD with no CP insertion can achieve significant performance improvement while reducing the computational complexity compared to the conventional QRM-MLBD with CP insertion.

The rest of the paper is organized as follows. Section 2 describes the iterative overlap QRM-MLBD for SC-MIMO spatial multiplexing with no CP insertion. Simulation results are presented in Sect. 3. The achievable throughput performance with the iterative overlap 
QRM-MLBD is compared with the conventional QRM-MLBD with CP insertion and the computational complexity of the iterative overlap QRM-MLBD is discussed as well. Finally, we conclude the paper in Sect. 4.

\section{Iterative Overlap QRM-MLBD}

\subsection{Transmission System Model}

Figure 1 illustrates the transmitter/receiver structure of SC-MIMO spatial multiplexing using the iterative overlap QRM-MLBD, where the numbers of transmit antennas and receive antennas are denoted by $N_{t}$ and $N_{r}$, respectively. At the transmitter, the information bit sequence is transformed into a data-modulated symbol sequence and then, serial-to-parallel (S/P) converted to $N_{t}$ parallel symbol sequences and each symbol sequence is transmitted from a different antenna.

The transmitted symbol sequence of each transmit antenna propagates through different channel and received by $N_{r}$ receive antennas at the receiver. The received symbol sequence on each receive antenna is divided into a sequence of blocks of $X$ symbols each. To detect an $N_{c}$-symbol block including the $X$-symbol block of interest at the beginning, block signal processing is applied to an extended block of $N_{c}+L-1$ symbols (referred to as observation window).

Iterative processing is applied to reduce the residual IBI. In the $i$ th iteration stage, the replica of IBI from the previous block is generated by using the $i$ th stage decision of the previous block. The replica of IBI from the next block is generated by the $(i-1)$ th stage decision of the next block. The IBIs are removed by subtracting their replicas from the received symbol sequence of interest over the observation window before applying QRM-MLBD. The received symbol sequence over the observation window at each receive antenna after IBI

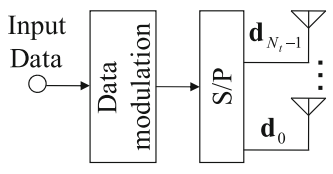

(a) Transmitter

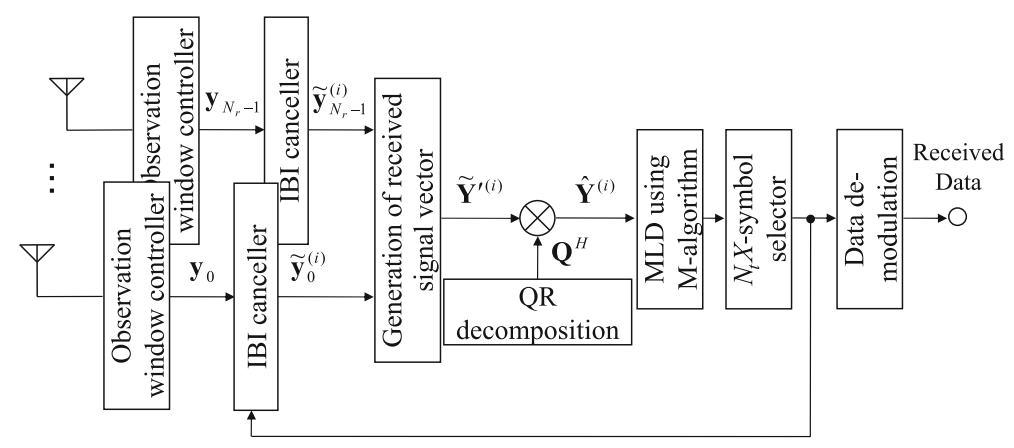

(b) Receiver

Fig. 1 Transmission system model. a Transmitter. b Receiver 


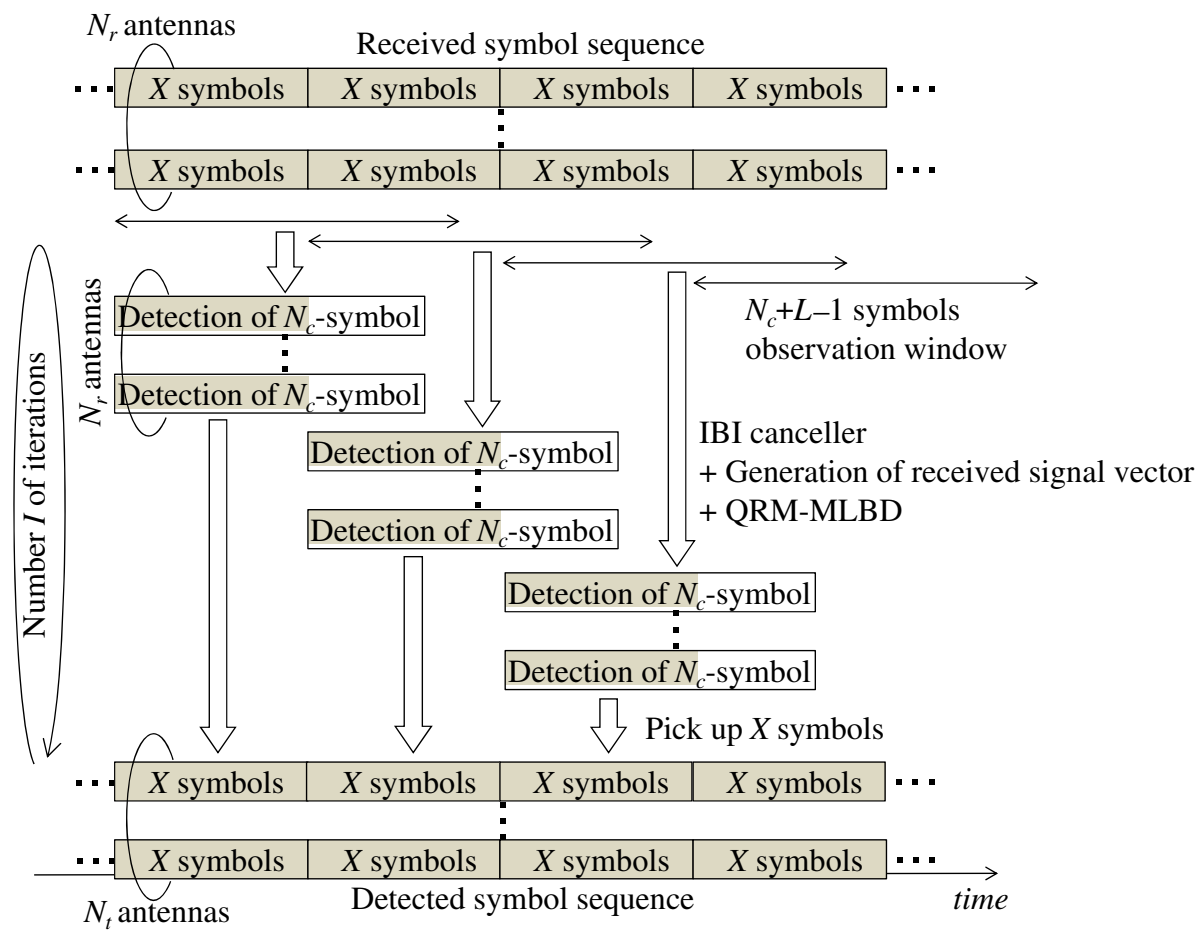

Fig. 2 Iterative overlap QRM-MLBD

suppression is represented by a column vector of $N_{c}+L-1$ elements. $N_{r}$ received symbol vectors are stacked to form a stacked received symbol vector. Then, by appropriately modifying the stacked received symbol vector, QRM-MLBD is applied to detect $N_{t}$ transmitted blocks of $N_{c}$ symbols each. After QRM-MLBD, the first $X$-symbol block is picked up for each transmit antenna from each detected block of $N_{c}$ symbols.

To detect the next $X$-symbol block, the observation window is shifted by $X$ symbols as shown in Fig. 2. By repeating this process, the continuously transmitted symbol sequence from each transmit antenna is detected. The above overlap QRM-MLBD processing is repeated $I$ times ( $I=0$ represents the initial iteration stage) to suppress the residual IBI sufficiently.

\subsection{Received Signal Representation}

A frequency-selective fading channel is assumed to be composed of symbol-spaced $L$ distinct propagation paths with different time delays. The channel impulse response between the $n_{t}$ th transmit antenna and the $n_{r}$ th receive antenna $h_{n_{r}, n_{t}}(\tau)$ is expressed as

$$
h_{n_{r}, n_{t}}(\tau)=\sum_{l=0}^{L-1} h_{n_{r}, n_{t}, l} \delta\left(\tau-\tau_{n_{r}, n_{t}, l}\right),
$$

where $h_{n_{r}, n_{t}, l}$ and $\tau_{n_{r}, n_{t}, l}$ are respectively the complex-valued path gain with $E\left[\left|h_{n_{r}, n_{t}, l}\right|^{2}\right]=1$ and the time delay of the $l$ th path between the $n_{t}$ th transmit antenna and the $n_{r}$ th receive antenna. The received $N_{c}+L-1$-symbol sequence, $\mathbf{y}_{n_{r}}=\left[y_{n_{r}}(0), \ldots, y_{n_{r}}(t), \ldots, y_{n_{r}}\left(N_{c}+\right.\right.$ 
$L-2)]^{T}$ with (.) ${ }^{T}$ denoting the transpose operation, over the observation window on the $n_{r}$ th receive antenna can be expressed using the matrix form as

$$
\begin{aligned}
\mathbf{y}_{n_{r}}= & \sqrt{\frac{2 E_{s}}{T_{s} N_{t}}} \sum_{n_{t}=0}^{N_{t}-1} \mathbf{h}_{n_{r}, n_{t}} \mathbf{d}_{n_{t}}+\sqrt{\frac{2 E_{S}}{T_{S} N_{t}}} \sum_{n_{t}=0}^{N_{t}-1} \mathbf{h}_{n_{r}, n_{t},-1} \mathbf{d}_{n_{t},-1} \\
& +\sqrt{\frac{2 E_{s}}{T_{S} N_{t}}} \sum_{n_{t}=0}^{N_{t}-1} \mathbf{h}_{n_{r}, n_{t},+1} \mathbf{d}_{n_{t},+1}+\mathbf{n}_{n_{r}},
\end{aligned}
$$

where $E_{s}$ and $T_{s}$ are respectively the symbol energy and duration. $\mathbf{d}_{n_{t}}=\left[d_{n_{t}}(0), \ldots, d_{n_{t}}\right.$ $\left.(t), \ldots, d_{n_{t}}\left(N_{c}-1\right)\right]^{T}$ represents the transmit symbol vector of interest from the $n_{t}$ th transmit antenna. $\mathbf{d}_{n_{t},-1(+1)}=\left[d_{n_{t},-1(+1)}(0), \ldots, d_{n_{t},-1(+1)}(t), \ldots, d_{n_{t},-1(+1)}\left(N_{c}-1\right)\right]^{T}$ represents the previous (next) transmit symbol vector. The first term of (2) denotes the desired signal and the second and third terms denote the IBI from the previous and the next blocks, respectively. $\mathbf{n}_{n_{r}}=\left[n_{n_{r}}(0), \ldots, n_{n_{r}}(t), \ldots, n_{n_{r}}\left(N_{c}+L-2\right)\right]^{T}$ is the zero-mean additive white Gaussian noise (AWGN) vector with the one-sided power spectrum density $N_{0}$. $\mathbf{h}_{n_{r}, n_{t}}, \mathbf{h}_{n_{r}, n_{t},-1}$, and $\mathbf{h}_{n_{r}, n_{t},+1}$ are respectively $\left(N_{c}+L-1\right) \times N_{c}$ channel impulse response matrixes between the $n_{t}$ th transmit antenna and the $n_{r}$ th receive antenna, given as

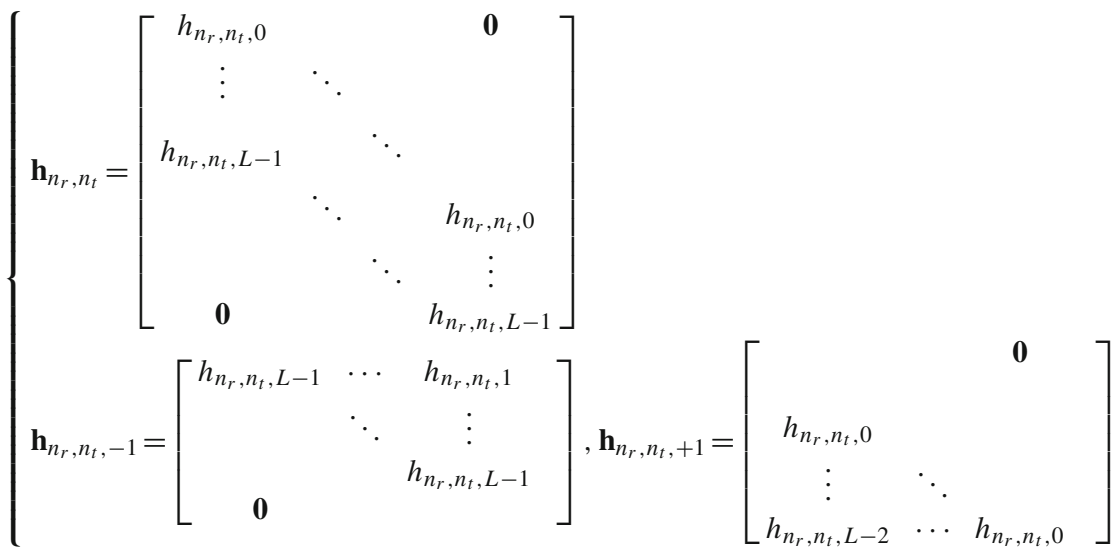

\subsection{Iterative Overlap QRM-MLBD}

\subsubsection{Stacked Received Symbol Vector}

In the $i$ th iteration stage, the IBI replica from the previous block is generated by using the decision of $\hat{\mathbf{d}}_{n_{t},-1}^{(i)}=\left[\hat{d}_{n_{t},-1}^{(i)}(0), \ldots, \hat{d}_{n_{t},-1}^{(i)}(t), \ldots, \hat{d}_{n_{t},-1}^{(i)}\left(N_{c}-1\right)\right]^{T}, n_{t}=0 \sim N_{t}-1$, of the previous block. When $i \geq 1$, the IBI replica from the next block is also generated by using the decision of $\hat{\mathbf{d}}_{n_{t},+1}^{(i-1)}=\left[\hat{d}_{n_{t},+1}^{(i-1)}(0), \ldots, \hat{d}_{n_{t},+1}^{(i-1)}(t), \ldots, \hat{d}_{n_{t},+1}^{(i-1)}\left(N_{c}-1\right)\right]^{T}, n_{t}=0 \sim N_{t}-1$, of the next block. The IBI cancellation is performed by subtracting the IBI replicas from the received signal as

$$
\tilde{\mathbf{y}}_{n_{r}}^{(i)}=\mathbf{y}_{n_{r}}-\left(\sqrt{\frac{2 E_{s}}{T_{S} N_{t}}} \sum_{n=0}^{N_{t}-1} \mathbf{h}_{n_{r}, n_{t},-1} \hat{\mathbf{d}}_{n_{t},-1}^{(i)}+\sqrt{\frac{2 E_{s}}{T_{s} N_{t}}} \sum_{n=0}^{N_{t}-1} \mathbf{h}_{n_{r}, n_{t},+1} \hat{\mathbf{d}}_{n_{t},+1}^{(i-1)}\right) .
$$


The received symbol vector after the IBI cancellation at each receive antenna is stacked to form an $N_{r}\left(N_{c}+L-1\right) \times 1$ stacked received symbol vector $\tilde{\mathbf{Y}}^{(i)}=\left[\left\{\tilde{\mathbf{y}}_{0}^{(i)}\right\}^{T} \cdots\left\{\tilde{\mathbf{y}}_{N_{r}-1}^{(i)}\right\}^{T}\right]^{T}$ as

$$
\begin{aligned}
& \tilde{\mathbf{Y}}^{(i)}=\left[\begin{array}{lll}
\left\{\tilde{\mathbf{y}}_{0}^{(i)}\right\}^{T} & \cdots & \left\{\tilde{\mathbf{y}}_{N_{r}-1}^{(i)}\right\}^{T}
\end{array}\right]^{T} \\
& =\sqrt{\frac{2 E_{S}}{T_{S} N_{t}}}\left[\begin{array}{ccc}
\mathbf{h}_{0,0} & \cdots & \mathbf{h}_{0, N_{t}-1} \\
\vdots & \ddots & \vdots \\
\mathbf{h}_{N_{r}-1,0} & \cdots & \mathbf{h}_{N_{r}-1, N_{t}-1}
\end{array}\right]\left[\begin{array}{c}
\mathbf{d}_{0} \\
\vdots \\
\mathbf{d}_{N_{t}-1}
\end{array}\right]+\left[\begin{array}{c}
\mathbf{n}_{0} \\
\vdots \\
\mathbf{n}_{N_{r}-1}
\end{array}\right] \\
& +\sqrt{\frac{2 E_{S}}{T_{S} N_{t}}}\left[\begin{array}{ccc}
\mathbf{h}_{0,0,-1} & \cdots & \mathbf{h}_{0, N_{t}-1,-1} \\
\vdots & \ddots & \vdots \\
\mathbf{h}_{N_{r}-1,0,-1} & \cdots & \mathbf{h}_{N_{r}-1, N_{t}-1,-1}
\end{array}\right]\left[\begin{array}{c}
\mathbf{d}_{0,-1}-\hat{\mathbf{d}}_{0,-1}^{(i)} \\
\vdots \\
\mathbf{d}_{N_{t}-1,-1}-\hat{\mathbf{d}}_{N_{t}-1,-1}^{(i)}
\end{array}\right] \\
& +\sqrt{\frac{2 E_{s}}{T_{S} N_{t}}}\left[\begin{array}{ccc}
\mathbf{h}_{0,0,+1} & \cdots & \mathbf{h}_{0, N_{t}-1,+1} \\
\vdots & \ddots & \vdots \\
\mathbf{h}_{N_{r}-1,0,+1} & \cdots & \mathbf{h}_{N_{r}-1, N_{t}-1,+1}
\end{array}\right]\left[\begin{array}{c}
\mathbf{d}_{0,+1}-\hat{\mathbf{d}}_{0,+1}^{(i-1)} \\
\vdots \\
\mathbf{d}_{N_{t}-1,+1}-\hat{\mathbf{d}}_{N_{t}-1,+1}^{(i-1)}
\end{array}\right] \\
& =\sqrt{\frac{2 E_{s}}{T_{S} N_{t}}} \mathbf{H D}+\sqrt{\frac{2 E_{s}}{T_{S} N_{t}}} \mathbf{H}_{-1}\left(\mathbf{D}_{-1}-\hat{\mathbf{D}}_{-1}^{(i)}\right)+\sqrt{\frac{2 E_{s}}{T_{S} N_{t}}} \mathbf{H}_{+1}\left(\mathbf{D}_{+1}-\hat{\mathbf{D}}_{+1}^{(i-1)}\right)+\mathbf{N},
\end{aligned}
$$

where $\mathbf{N}=\left[\begin{array}{lll}\left\{\mathbf{n}_{0}\right\}^{T} & \cdots & \left\{\mathbf{n}_{N_{r}-1}\right\}^{T}\end{array}\right]^{T}$ is the $N_{r}\left(N_{c}+L-1\right) \times 1$ stacked noise vector.

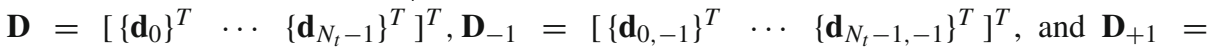
$\left[\left\{\mathbf{d}_{0,+1}\right\}^{T} \ldots \quad\left\{\mathbf{d}_{N_{t}-1,+1}\right\}^{T}\right]^{T}$ are the $N_{t} N_{c} \times 1$ stacked transmit symbol vectors. $\mathbf{H}, \mathbf{H}_{-1}$, and $\mathbf{H}_{+1}$ are equivalent channel matrixes of size $N_{r}\left(N_{c}+L-1\right) \times N_{t} N_{c}$, which represent space and time-domain channel, given by

$$
\left\{\begin{array}{l}
\mathbf{H}=\left[\begin{array}{ccc}
\mathbf{h}_{0,0} & \cdots & \mathbf{h}_{0, N_{t}-1} \\
\vdots & \ddots & \vdots \\
\mathbf{h}_{N_{r}-1,0} & \cdots & \mathbf{h}_{N_{r}-1, N_{t}-1}
\end{array}\right] \\
\mathbf{H}_{-1}=\left[\begin{array}{ccc}
\mathbf{h}_{0,0,-1} & \cdots & \mathbf{h}_{0, N_{t}-1,-1} \\
\vdots & \ddots & \vdots \\
\mathbf{h}_{N_{r}-1,0,-1} & \cdots & \mathbf{h}_{N_{r}-1, N_{t}-1,-1}
\end{array}\right], \mathbf{H}_{+1}=\left[\begin{array}{ccc}
\mathbf{h}_{0,0,+1} & \cdots & \mathbf{h}_{0, N_{t}-1,+1} \\
\vdots & \ddots & \vdots \\
\mathbf{h}_{N_{r}-1,0,+1} & \cdots & \mathbf{h}_{N_{r}-1, N_{t}-1,+1}
\end{array}\right]
\end{array}\right.
$$

The second and the third terms of (5) are the residual IBIs from the previous and the next blocks, respectively.

\subsubsection{Modification of the Stacked Received Symbol Vector}

Overlap QRM-MLBD for SISO systems $[13,14]$ utilizes the property that the IBI from the next block, which cannot be removed in the initial iteration stage, exists only on the elements near the bottom of the received symbol vector. QRM-MLBD is applied to the received symbol vector and then, the residual IBI is significant near the end of $N_{c}$-symbol block after QRMMLBD. Therefore, symbol error rate near the beginning of the block is lower while symbol error rate near the end of the block is higher. Based on the above observation, overlap QRMMLBD can effectively suppress the IBI by picking up only the reliable first $X$-symbol block from the $N_{c}$-symbol block. 
However, in (5), the IBI from the next block exists on the elements near the bottom of received symbol vector at each receive antenna. Therefore, if QRM-MLBD is applied to (5) directly, the effect of IBI spreads over the all symbols in the entire block. To extend the previously proposed overlap QRM-MLBD to the MIMO systems, we modify the stacked received symbol vector as

$$
\tilde{\mathbf{Y}}^{\prime(i)}=\left[\left\{\tilde{\mathbf{Y}}^{(i)}(0)\right\}^{T}, \ldots,\left\{\tilde{\mathbf{Y}}^{(i)}(t)\right\}^{T}, \ldots,\left\{\tilde{\mathbf{Y}}^{(i)}\left(N_{c}-L-2\right)\right\}^{T}\right]^{T},
$$

where $\tilde{\mathbf{Y}}^{(i)}(t)=\left[\tilde{\mathbf{Y}}_{0}^{(i)}(t), \ldots, \tilde{\mathbf{Y}}_{N_{r}-1}^{(i)}(t)\right]^{T}$ denotes the $t$ th $N_{r} \times 1$ size received symbol vector after IBI cancellation. After the above modification, the equivalent channel matrixes corresponding to the IBIs from the previous and the next blocks are permutated as

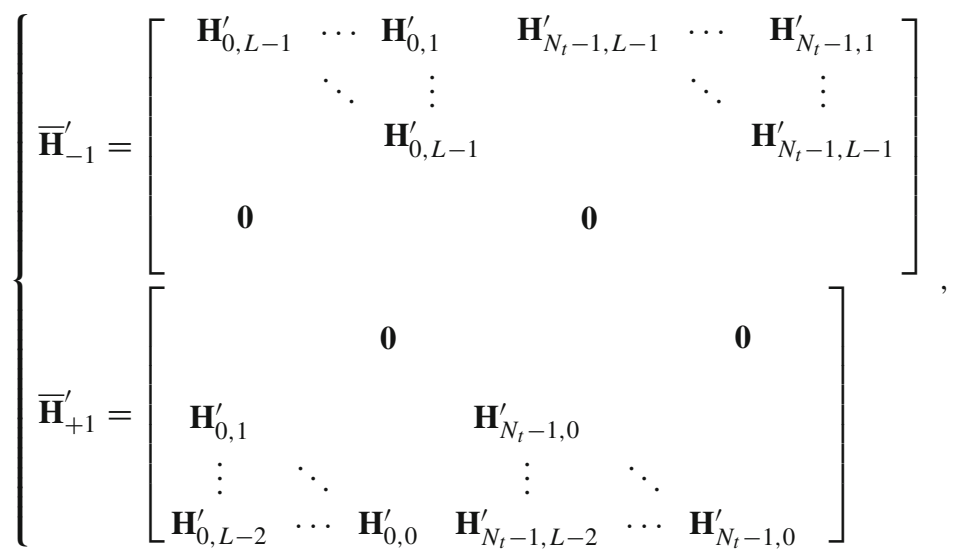

where $\mathbf{H}_{n_{t}, l}^{\prime}=\left[h_{0, n_{t}, l} \ldots, h_{N_{r}-1, n_{t}, l}\right]^{T}$. It can be seen from (7) and (8) that the IBI from the next block exists only in the elements near the bottom of the modified stacked received symbol vector.

In QRM-MLBD, M-algorithm [15] is performed starting from the last symbol in the stacked transmit symbol vector. Since overlap QRM-MLBD outputs only $X$-symbol block for each transmit antenna which suffers less of the IBI from the next symbol block, the $N_{t} N_{c}$ symbols in the stacked transmit symbol vector $\mathbf{D}$ is changed as

$$
\mathbf{D}^{\prime}=\left[\mathbf{D}^{T}\left(N_{c}-1\right), \ldots, \mathbf{D}^{T}(t), \ldots, \mathbf{D}^{T}(0)\right]^{T},
$$

where $\mathbf{D}(t)=\left[d_{N_{t}-1}(t), \ldots, d_{0}(t)\right]^{T}$ denotes the $t$ th $N_{t} \times 1$ size transmit symbol vector. By the above modification of the stacked received symbol vector and ordering of the stacked transmit symbol vector, the equivalent channel matrix for the desired signal component $\mathbf{H}$ is given as

$$
\overline{\mathbf{H}}^{\prime \prime}=\left[\begin{array}{cccc}
\mathbf{0} & & & \mathbf{H}_{L-1}^{\prime \prime} \\
& & \therefore & \vdots \\
\mathbf{H}^{\prime \prime}{ }_{L-1} & \therefore & & \mathbf{H}_{0}^{\prime \prime} \\
\vdots & & \therefore & \\
\mathbf{H}^{\prime \prime}{ }_{0} & & &
\end{array}\right],
$$


where

$$
\mathbf{H}^{\prime \prime}=\left[\begin{array}{ccc}
h_{0, N_{t}-1, l} & \cdots & h_{0,0, l} \\
\vdots & \ddots & \vdots \\
h_{N_{r}-1, N_{t}-1, l} & \cdots & h_{N_{r}-1,0, l}
\end{array}\right] .
$$

It can be seen from (7), (10), and (11) that the modified stacked received symbol vector is similar to the SISO case [14] as shown in Fig. 3. Therefore, the previously proposed overlap QRM-MLBD can be applied in a similar way to the MIMO system.

\subsubsection{QRM-MLBD}

QR decomposition is applied to the equivalent channel matrix $\overline{\mathbf{H}}^{\prime \prime}$ to obtain $\overline{\mathbf{H}}^{\prime \prime}=\mathbf{Q R}$, where $\mathbf{Q}$ is an $N_{r}\left(N_{c}+L-1\right) \times N_{t} N_{c}$ unitary matrix and $\mathbf{R}$ is an $N_{t} N_{c} \times N_{t} N_{c}$ upper triangular matrix. Since the equivalent channel matrix for the desired signal component $\overline{\mathbf{H}}^{\prime \prime}$ is shown in (10), the unitary matrix $\mathbf{Q}$ and the upper triangular matrix $\mathbf{R}$ obtained by $\mathrm{QR}$ decomposition of $\overline{\mathbf{H}}^{\prime \prime}$ are represented as

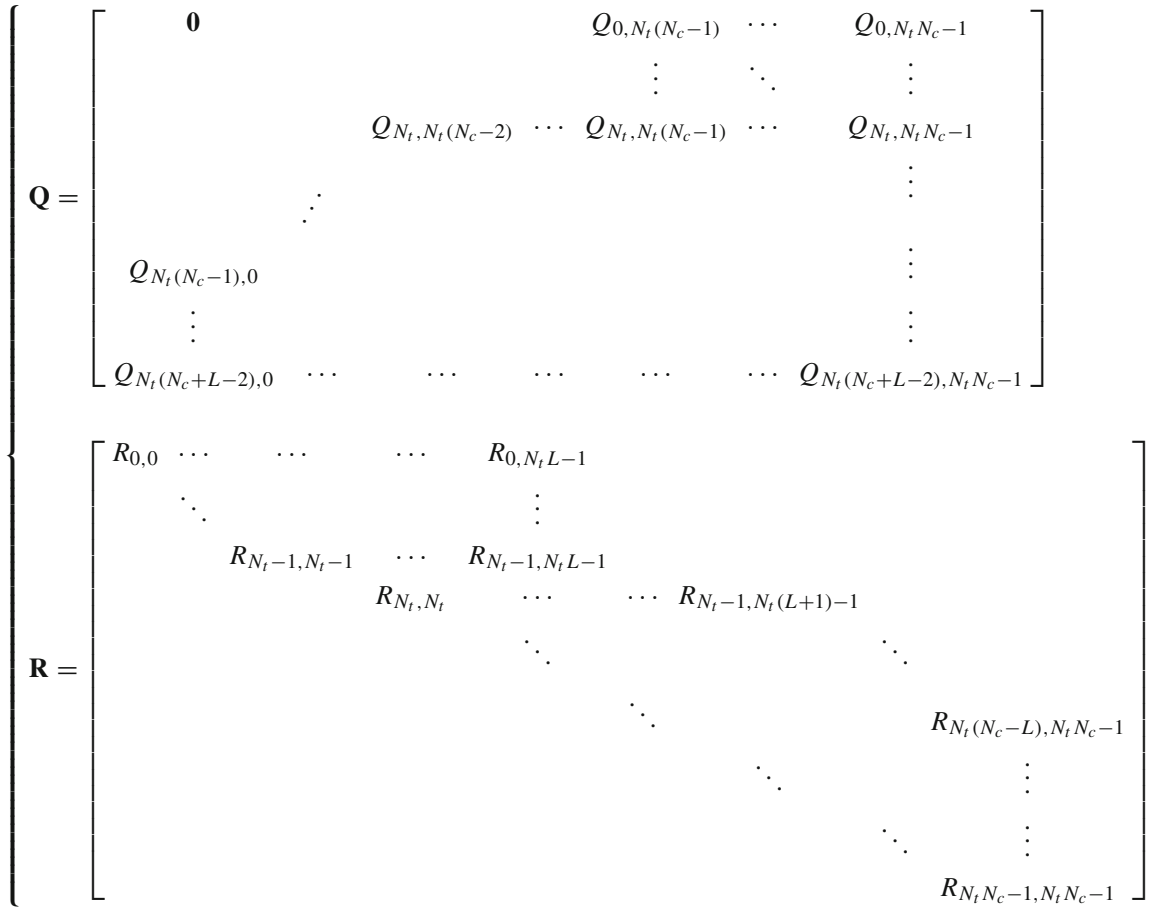

The transformed received signal vector $\hat{\mathbf{Y}}^{(i)}$ is obtained as

$$
\begin{aligned}
\hat{\mathbf{Y}}^{(i)}= & \mathbf{Q}^{H} \tilde{\mathbf{Y}}^{\prime(i)}=\sqrt{\frac{2 E_{s}}{T_{s} N_{t}}} \mathbf{R} \mathbf{D}^{\prime}+\sqrt{\frac{2 E_{s}}{T_{s} N_{t}}} \hat{\mathbf{H}}_{-1}\left(\mathbf{D}_{-1}-\hat{\mathbf{D}}_{-1}^{(i)}\right) \\
& +\sqrt{\frac{2 E_{s}}{T_{s} N_{t}}} \hat{\mathbf{H}}_{+1}\left(\mathbf{D}_{+1}-\hat{\mathbf{D}}_{+1}^{(i-1)}\right)+\hat{\mathbf{N}}
\end{aligned}
$$




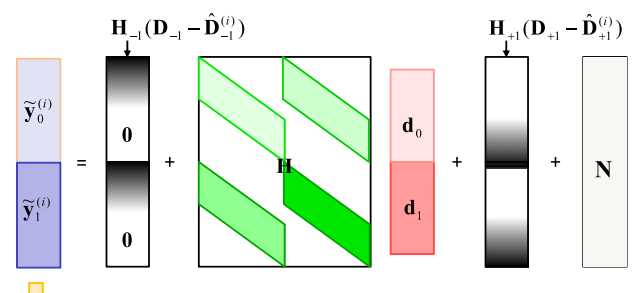

Modification of the stacked received signal vector
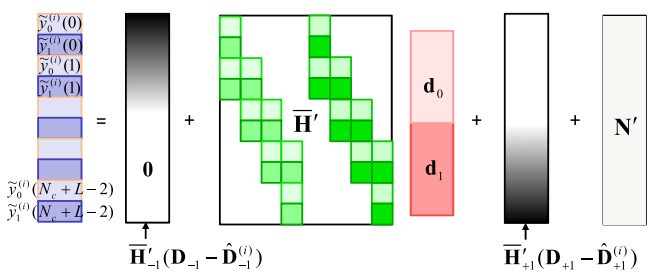

Ordering of the stacked transmit symbol vector
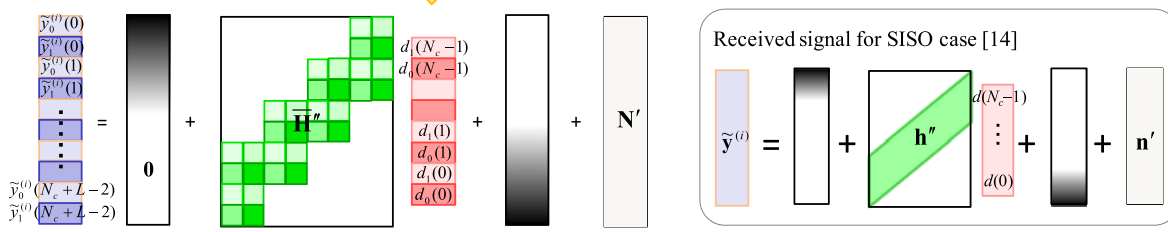

Fig. 3 Modified received signal vector $\left(N_{t}=N_{r}=2\right)$

where $\hat{\mathbf{H}}_{-1}=\mathbf{Q}^{H} \overline{\mathbf{H}}_{-1}^{\prime}, \quad \hat{\mathbf{H}}_{+1}=\mathbf{Q}^{H} \overline{\mathbf{H}}_{+1}^{\prime}, \quad \hat{\mathbf{N}}=\mathbf{Q}^{H} \mathbf{N}$, and (.) ${ }^{H}$ denotes the Hermitian transpose operation.

From (13), the ML solution is to select the path with the minimum Euclidean distance in a $N_{t} N_{c}$-stages tree diagram. This can be realized by M-algorithm. At each stage, the best $M$ surviving paths selected from all the paths are passed to the next stage. The squared Euclidean distance is used for branch metric calculation. Data demodulation is carried out by tracing back the path having the smallest path metric at the last stage. In this paper, the stopping criterion [14] can be applied similarly to the SISO case to stop the tree search at an earlier stage to reduce the detection complexity. It can be seen from (12) and (13) that the branch metric beyond the $\left(N_{t} L+n\right)$ th stage does not affect the detection of the $\left\lfloor n / N_{t}\right\rfloor$ th data symbol transmitted from the $\left(n \bmod N_{t}\right)$ th transmit antenna, where $\lfloor x\rfloor$ represents the largest integer smaller than or equal to $x$. Therefore, the tree search using M-algorithm can be stopped at the $\left(N_{t}(X+L)\right.$-1)th stage in order to output an $X$-symbol block for each transmit antenna.

\subsubsection{IBI power distribution}

Without CP insertion, the previous and the next blocks produce IBI. In the initial iteration stage $(i=0)$, IBI from the previous block can be suppressed by using the decision of the previous block as (5). However, IBI from the next block cannot be removed. Here, we consider the distribution of IBI from next block after multiplying $\mathbf{Q}^{H}$ [third term of (13)]. It can be seen from (12) that since $\mathbf{Q}^{H}$ is an unitary matrix, the absolute value of each element of $\mathbf{Q}^{H}$ is larger in upper row vector. Therefore, the absolute value of each element of $\hat{\mathbf{H}}_{+1}=\mathbf{Q}^{H} \overline{\mathbf{H}}_{+1}^{\prime}$ of (13) is larger in an upper row vector and is smaller in a lower row vector. As a result, the IBI power from the next block is more significant at an element closer 
Table 1 Computer simulation condition

\begin{tabular}{lll}
\hline Transmitter & Modulation & 16QAM \\
& Number of transmit antennas & $N_{t}=2$ \\
& Number of symbols per packet & $N_{t} N_{p}=384$ symbols \\
Channel & GI length & $N_{g}=16$ \\
& Fading type & Frequency-selective block Rayleigh \\
& Power delay profile & $L=16$-path uniform \\
Receiver & Time delay & $\tau_{l}=l(l=0 \sim L-1)$ \\
& Number of receive antennas & $N_{r}=2$ \\
& Channel estimation & Ideal \\
\hline
\end{tabular}

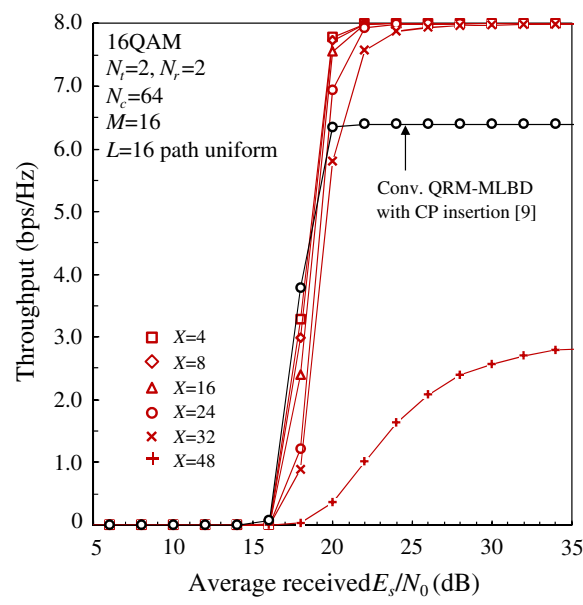

(a) $I=0$

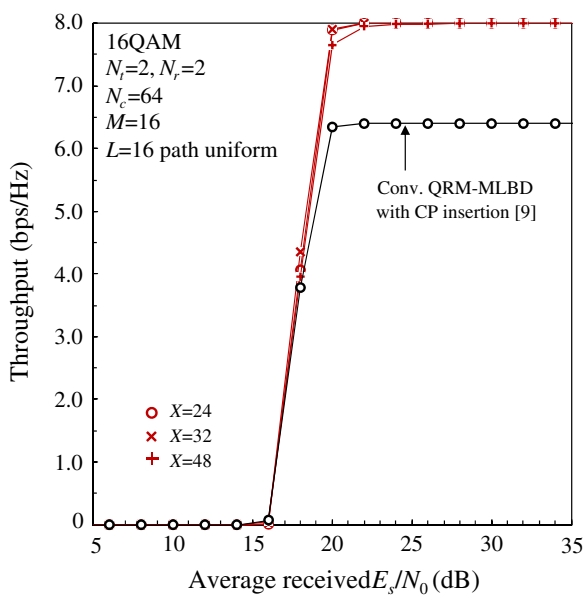

(b) $I=1$

Fig. 4 Throughput performance. a $I=0$. b $I=1$

to the beginning of the transformed signal vector while the IBI power from the next block is less significant at an element near the end of the transformed signal vector. Hence, in the $\mathrm{M}$-algorithm, the probability of erroneously removing the correct path is higher at last stages due to the stronger IBI while it is lower at early stages, resulting in higher error rate for the symbols near the end of the $N_{c}$-symbol block. Therefore, only the reliable $X$-symbol block at early stages is picked up from the detected block.

\section{Computer Simulation Results}

The performance of SC-MIMO spatial multiplexing using iterative overlap QRM-MLBD is evaluated by computer simulation. The simulation condition is summarized in Table 1 . 16QAM is used for data modulation. We assume $N_{t}=2, N_{r}=2$, and a frequency-selective quasi-static Rayleigh fading channel with an $L=16$-path uniform power delay profile. Ideal channel estimation is assumed. In this paper, SC-MIMO packet transmission is considered, where one packet is composed of 384 symbols. 


\subsection{Throughput Performance}

Figure 4 plots the throughput performance as a function of average received $E_{S} / N_{0}$ for $N_{c}=64, X=4 \sim 48, I=0$ and 1 , and $M=16 ; X$ is the number of symbols to be picked up and $M$ is the number of surviving paths in the M-algorithm. In this paper, the throughput is defined as $N_{t} \log _{2} Z \times(1-\mathrm{PER}) /\left(1+N_{g} / N_{c}\right)$, where $Z$ is the modulation level and PER denotes the packet error rate. The throughput performance of the conventional QRMMLBD with CP insertion is also plotted for comparison. The training sequence (TS) aided QRM-MLBD with TS length of 16 symbols [9] is used, which is similar to the conventional QRM-MLBD with CP insertion. It can be seen from Fig. 4 that iterative overlap QRM-MLBD improves the throughput performance when smaller $X$ is used. This is because at early stages of M-algorithm, the IBI from the next block is less significant. However, the use of smaller $X$ increases the detection complexity. It can also be seen that iterative processing improves the throughput even if large $X$ is used. With no iteration $(I=0), X=4$ should be used to improve the throughput performance sufficiently. However, when $I=1$, a much larger $X$ (e.g, $X=48$ ) can be used. Since iterative overlap QRM-MLBD does not require the CP insertion, the peak throughput is higher than that of the conventional QRM-MLBD with CP insertion.

\subsection{Computational Complexity}

The computational complexity of iterative overlap QRM-MLD is discussed. The computational complexity here is defined as the number of complex multiplications per symbol. The computational complexity for the iterative overlap QRM-MLBD and the conventional QRM-MLBD with CP insertion (TS-aided QRM-MLBD) are shown in Table 2. Time-domain signal processing is also implemented in TS-aided QRM-MLBD.

The overall computational complexity per symbol is shown in Fig. 5 as a function of the number of iterations $I$ for $N_{c}=64$ and $M=16$. As we mentioned in the previous section, $X=4$ should be used to sufficiently improve the throughput performance with no iteration $(I=0)$ and $X=48$ can be used when $I=1$. Therefore, $X$ is set to 4 and 48 for $I=0$ and 1 , respectively. When $I=2, X=48$ is also required. The overall computational complexity of the conventional QRM-MLBD with CP insertion is also plotted for reference. Since iterative processing improves the throughput even if large $X$ is used, the overall computational complexity can be reduced even if iterative processing is introduced when $N_{c}=64$.

In the next, we discuss the relationship between the observation window size $\left(N_{c}+L-1\right.$ symbols) and the overall computational complexity. Figure 6 plots the overall computational complexity as a function of $N_{c}$ when the best combination of $I$ and $X$ is used to achieve a throughput of $8 \mathrm{bps} / \mathrm{Hz}$ at $E_{S} / N_{0}=22 \mathrm{~dB}$. When a smaller $N_{c}$ is used, larger $I$ and smaller $X$ are needed. Therefore, the computational complexity of the IBI cancellation, computation

Table 2 Number of complex multiplications per symbol

\begin{tabular}{lll}
\hline & Iterative overlap QRM-MLBD & TS-aided QRM-MLBD [9] \\
\hline IBI cancellation & $(I+1) L(L-1) N_{r} / X$ & \\
QR decomposition & $N_{r}\left(N_{c}+L-1\right)\left(N_{t} N_{c}\right)^{2} / N_{p}$ & $N_{r} N_{t}^{2}\left(N_{c}+N_{g}\right) N_{c}^{2} / N_{p}$ \\
Computation of $\hat{\mathbf{Y}}^{(i)}$ & $(I+1) N_{r}\left(N_{c}+L-1\right) N_{c} / X$ & $N_{r}\left(N_{c}+N_{g}\right)$ \\
Path metric calc. & $(I+1)\left\{(1+M) N_{t}(X+L) Z\right.$ & $\left\{(1+M) N_{t} N_{c} Z+(1-M) Z\right.$ \\
& $+(1-M) Z+M N_{t}(X+L)$ & $\left.+M N_{t} N_{c}\left(N_{t} N_{c}-1\right) / 2\right\} / N_{t} N_{c}$ \\
& $\left.\left(N_{t}(X+L)-1\right) / 2\right\} / N_{t} X$ & \\
& &
\end{tabular}


Fig. 5 Impact of $I$ on the overall computational complexity

Fig. 6 Impact of $N_{c}$ on the overall computational complexity
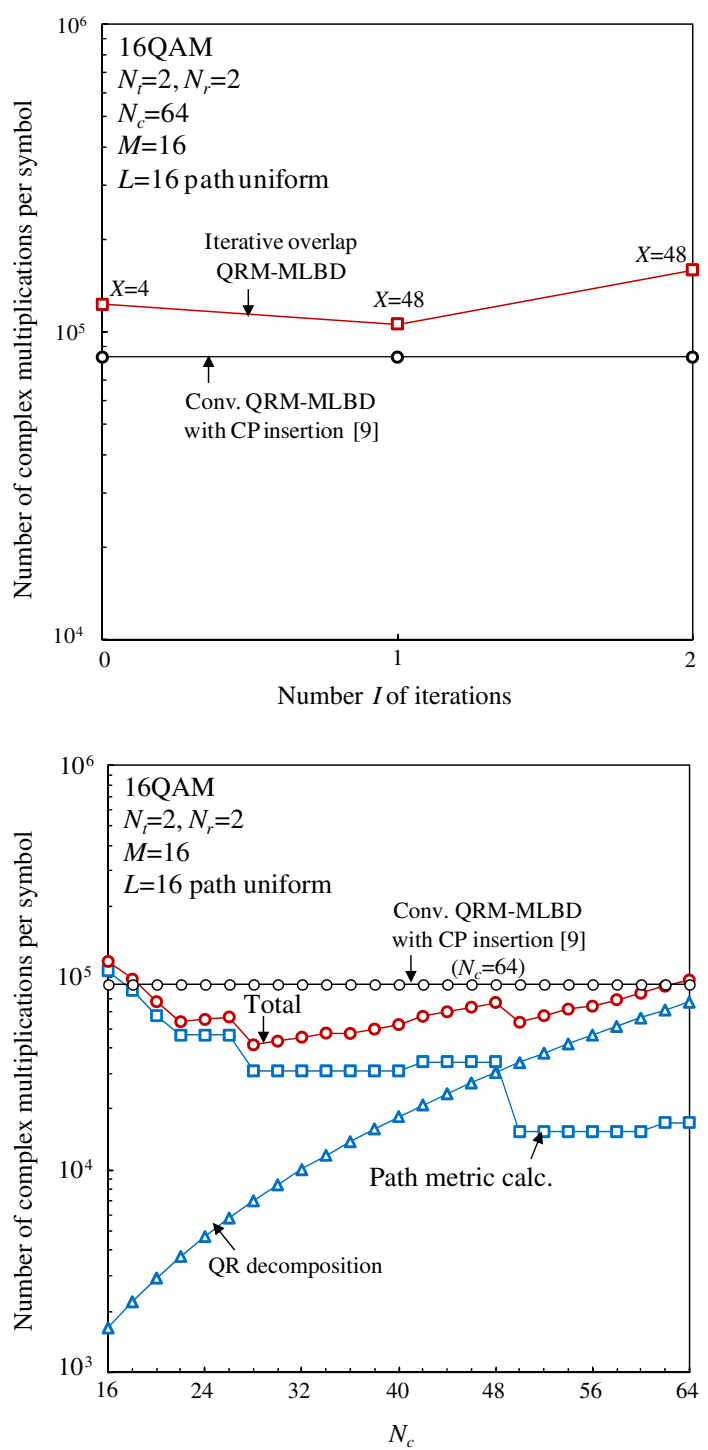

of $\hat{\mathbf{Y}}^{(i)}$ as well as path metric computation increases (because of larger $I$ and smaller $X$ ). On the other hand, since the size of the channel matrix is small (the size of equivalent channel matrix is $\left.N_{r}\left(N_{c}+L-1\right) \times N_{t} N_{c}\right)$, the computational complexity of QR decomposition reduces. It is understood from Fig. 6 that the overall computational complexity to achieve a peak throughput of $8 \mathrm{bps} / \mathrm{Hz}$ is lowest when $N_{c}=28$ and is about $50 \%$ of the conventional QRM-MLBD $\left(N_{c}=64\right)$ with CP insertion.

\section{Conclusion}

In this paper, we proposed a time-domain iterative overlap QRM-MLBD which requires no $\mathrm{CP}$ insertion for SC-MIMO spatial multiplexing. To extend our previously proposed iterative 
overlap QRM-MLBD to the MIMO systems, we introduce an appropriate modification of the stacked received symbol vector of SC-MIMO spatial multiplexing. Remembering that the IBI is significant near the bottom of the elements in the modified stacked received symbol vector and the residual IBI is significant near the end of the detected symbol block after QRM-MLBD, only the reliable $X$ symbols are picked up after performing QRM-MLBD. To detect a continuously transmitted symbol stream, the present observation window for performing QRM-MLBD is overlapped with the previous and the next observation windows. To further improve the detection performance, iterative processing was introduced. It has been showed that the proposed iterative overlap QRM-MLBD improves the throughput by $25 \%$ than the conventional QRM-MLBD with CP insertion $\left(N_{c}=64, N_{g}=16\right)$. The overall computational complexity can be reduced to $50 \%$ when compared with the conventional QRM-MLBD with CP insertion $\left(N_{c}=64\right)$.

Open Access This article is distributed under the terms of the Creative Commons Attribution License which permits any use, distribution, and reproduction in any medium, provided the original author(s) and the source are credited.

\section{References}

1. Foschini, G. J., \& Gans, M. J. (1998). On limits of wireless communications in a fading environment when using multiple antennas. Wireless Personal Communications, 6(3), 311-335.

2. Ekstrom, H., Furuskar, A., Karlsson, J., Meyer, M., Parkvall, S., Torsner, J., et al. (2006). Technical solutions for the 3G long-term evolution. IEEE Communications Magazine, 44(3), 38-45.

3. Benjamin, N., Chan-Tong, L., \& Falconer, D. (2007). Turbo frequency domain equalization for singlecarrier broadband wireless systems. EEE Transactions on Wireless Communications, 6(2), 759-767.

4. Higuchi, K., Kawai, H., Maeda, N., Taoka, H., \& Sawahashi, M. (2006). Experiments on real-time 1-Gb/s packet transmission using MLD-based signal detection in MIMO-OFDM broadband radio access. IEEE Journal on Selected Areas Communications, 24(6), 1141-1153.

5. 3GPP, TS 36.211 (V10.4.0) (2011). 3rd Generation partnership project (3GPP); evolved universal terrestrial radio access (E-UTRA); physical channels and modulation (release 10).

6. Proakis, J. G., \& Salehi, M. (2008). Digital communications (5th ed.). New York: McGraw-Hill.

7. Nakajima, A., Garg, D., \& Adachi, F. (2005). Throughput of turbo coded hybrid ARQ using single-carrier MIMO multiplexing. In Proceedings of IEEE 61st vehicular technology conference (VTC2005-Spring) (Vol. 1, pp. 610-614). Stockholm, Sweden, 30 May-1 June.

8. Nagatomi, K., Higuchi, K., \& Kawai, H. (2009). Complexity reduced MLD based on QR decomposition in OFDM MIMO multiplexing with frequency domain spreading and code multiplexing. In Proceedings of IEEE wireless communications and networking conference (WCNC 2009). Budapest, Hungary, 5-8 Apr.

9. Yamamoto, T., Takeda, K., \& Adachi, F. (2011). Training sequence-aided QRM-MLD block signal detection for single-carrier MIMO spatial multiplexing. In Proceedings of 2011 IEEE international conference on communications (ICC 2011). Kyoto, Japan, 5-9 June.

10. Martoyo, I., Weiss, T., Capar, F., \& Jondral, F. K. (2003). Low complexity CDMA downlink receiver based on frequency domain equalization. In Proceedings of IEEE 58th vehicular technology conference (VTC2003-Fall). Orlando, Florida, USA, 6-9 Sept.

11. Obara, T., Takeda, K., Lee, K., \& Adachi, F. (2011). Performance comparison of overlap FDE and sliding-window chip equalization for multi-code DS-CDMA in a frequency-selective fading channel. IEICE Transactions on Communications, E94-B(3), 750-757.

12. Ishihara, K., Takatori, Y., Kubota, S., \& Adachi, F. (2009). Multiuser detection for asynchronous broadband single-carrier transmission systems. IEEE Transactions on Vehicular Technology, 58(6), 3055-3071.

13. Moroga, H., Yamamoto, T., \& Adachi, F. (2012). Overlap QRM-ML block signal detection for singlecarrier transmission without $\mathrm{CP}$ insertion. In Proceedings of IEEE 75th vehicular tecnnology conference (VTC2012-Spring). Yokohama, Japan, 6-9 May. 
14. Moroga, H., Yamamoto, T., \& Adachi, F. (2012). Iterative overlap TD-QRM-ML block signal detection for single-carrier transmission without $\mathrm{CP}$ insertion. In Proceedings of IEEE 76th vehicular technology conference (VTC2012-Fall). Québec City, Canada, 3-6 Sept.

15. Anderson, J. B., \& Mohan, S. (1984). Sequential coding algorithms: A survey and cost analysis. IEEE Transactions on Communications, 32(2), 169-176.
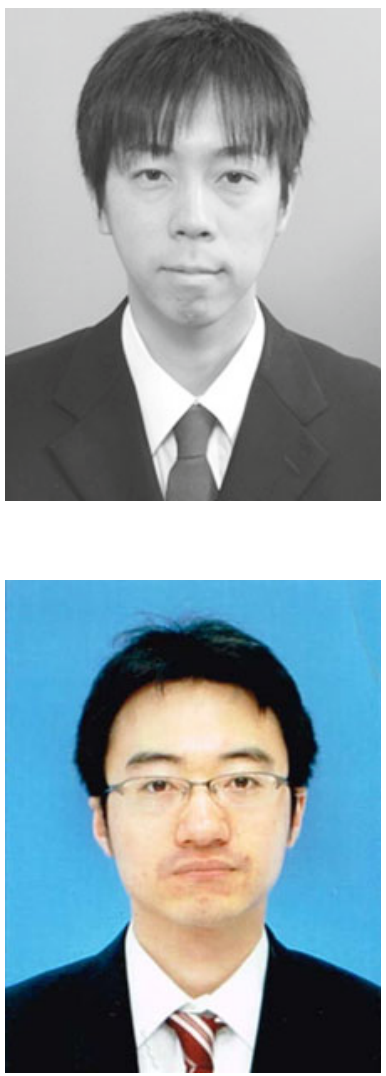

Hideyuki Moroga received his B.S. degree in Information System Engineering from Kanazawa University, Kanazawa, Japan, in 2010 and M.S. degree in communications engineering, from Tohoku University, Sendai, Japan, in 2012. Currently he is with NTT DoCoMo. His research interests include time-domain equalization and signal detection techniques for mobile communication systems.

Tetsuya Yamamoto received his B.S. degree in Electrical, Information and Physics Engineering in 2008 and M.S. and Dr. Eng. degrees in communications engineering from Tohoku University, Sendai Japan, in 2010 and 2012, respectively. From April 2010 to March 2013, he was a Japan Society for the Promotion of Science (JSPS) research fellow. Since April 2013, he has been with Panasonic Corporation. He was a recipient of the 2008 IEICE RCS (Radio Communication Systems) Active Research Award and Ericsson Best Student Award 2012. 


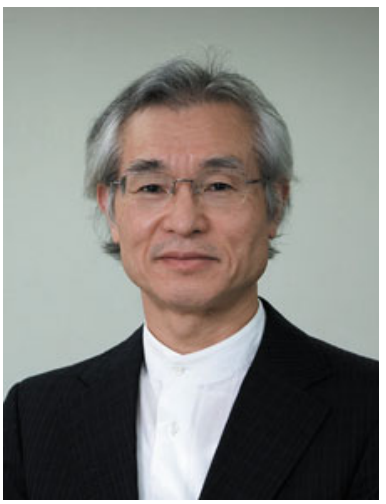

Fumiyuki Adachi received the B.S. and Dr. Eng. degrees in electrical engineering from Tohoku University, Sendai, Japan, in 1973 and 1984, respectively. In April 1973, he joined the Electrical Communications Laboratories of Nippon Telegraph \& Telephone Corporation (now NTT) and conducted various types of research related to digital cellular mobile communications. From July 1992 to December 1999, he was with NTT Mobile Communications Network, Inc. (now NTT DoCoMo, Inc.), where he led a research group on wideband/broadband CDMA wireless access for IMT-2000 and beyond. Since January 2000, he has been with Tohoku University, Sendai, Japan, where he is a Professor of Communications Engineering at the Graduate School of Engineering. In 2011, he was appointed a Distinguished Professor. His research interest is in the areas of wireless signal processing and networking including broadband wireless access, equalization, transmit/receive antenna diversity, MIMO, adaptive transmission, channel coding, etc. From October 1984 to September 1985, he was a United Kingdom SERC Visiting Research Fellow in the Department of Electrical Engineering and Electronics at Liverpool University. Dr. Adachi is an IEEE Fellow and a VTS Distinguished Lecturer for 2011 to 2013. He was a co-recipient of the IEEE Vehicular Technology Transactions Best Paper of the Year Award 1980 and again 1990 and also a recipient of Avant Garde award 2000. He is a Fellow of Institute of Electronics, Information and Communication Engineers of Japan (IEICE) and is a recipient of IEICE Achievement Award 2002 and a co-recipient of the IEICE Transactions Best Paper of the Year Award 1996, 1998 and again 2009. He is a recipient of Thomson Scientific Research Front Award 2004, Ericsson Telecommunications Award 2008, Telecom System Technology Award 2009, and Prime Minister Invention Prize 2010, British Royal Academy of Engineering Distinguished Visiting Fellowship 2011, and KDDI Foundation Research Award 2012. 